\title{
DIPTERES TORRENTICOLES PEU CONNUS. VIII. LES ATHERICIDAE (IBISIA VAILLANTI N. SP.) DU SUD DE LA FRANCE (BRACHYCERA, ORTHORRHAPHA)
}

\author{
par A. G. B. THOMAS ${ }^{1}$
}

L'imago ơ d'Ibisia vaillanti n. sp., proche d'I. marginata (Fabricius) est décrite et figurée. Cette espèce a été rencontrée dans le Sud de la France et en Espagne.

\section{Poorly known torrential Diptera. VIII. Athericidae (lbisia vaillanti $n$. sp.) from South of France (Brachycera, Orthorrhapha)}

The male adult of Ibisia vaillanti $\mathrm{n}$. sp., similar to that of $I$. marginata (Fabricius) is described and illustrated. This species has been found in the south of France and Spain.

La prospection de nombreux cours d'eau, à des altitudes inférieures à $1000 \mathrm{~m}$, a permis la récolte d'imagos d'Athericidae d'une espèce nouvelle, très voisine d'Ibisia marginata (Fabricius, 1781). Les deux espèces se distinguent essentiellement par la morphologie des genitalia.

\section{Description d'lbisia vaillanti n. sp.}

IMAGo ô (en alcool).

Tête.

Brun noirâtre. Yeux rapprochés mais ne se touchant pas, à reflets très nettement verts. Triangle ocellaire peu proéminent. Antennes brunes dont les articles 1 et 2 portent de fortes soies noires. Joues et front à longue et dense pilosité noire. Clypeus brun foncé hémisphérique et très saillant. Palpes brun noirâtre, à forte pilosité noire, paraissant légèrement plus longs et plus minces que chez $I$. marginata, mais aussi un peu plus fortement incurvés (presque à angle droit). Trompe brune, longue et volumineuse, orientée selon l'axe dorso-

1. Laboratoire d'Hydrobiologie (ERA 702), Université Paul-Sabatier, 118, route de Narbonne, 31062 Toulouse Cedex. 
ventral de la tête. Moitié postéro-inférieure de la tête à longue et fine pilosité blanche ou brune; moitié supérieure portant des soies plus courtes, plus épaisses et noires.

\section{Thorax.}

Mesonotum brun noirâtre brillant, scutellum de même couleur mais à pilosité plus longue, noire ou blanche selon les exemplaires. Pleures, coxas et pattes brun foncé. Les pattes peuvent être proportionnellement plus épaisses que chez I. marginata: sur les spécimens d'I. vaillanti provenant du Rio Tambre (Espagne) où pullule Atrichops crassipes, tibia 3 et tarse 3 sont très sensiblement épaissis, tout comme chez cette dernière espèce. Ailes plus foncées, dans l'ensemble, que chez $I$. marginata, en particulier dans la région apicale et la cellule basale postérieure (fig. 1). Balanciers brun grisâtre foncé.

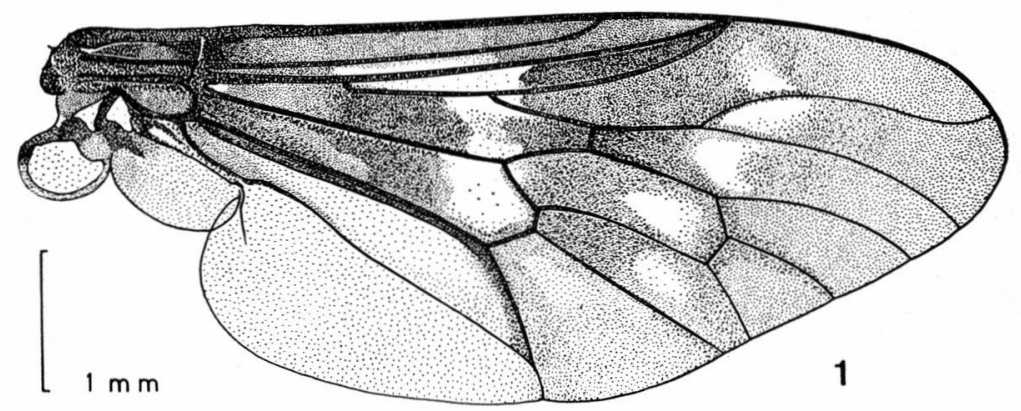

FIG. 1. - Ibisia vaillanti : aile.

\section{Abdomen.}

Brun noirâtre à pilosité : blanche, longue et dense ventralement, de couleur variable et plus courte dorsalement.

Sur les exemplaires conservés à sec, un poudré gris recouvre :

- sur la tête : essentiellement le clypeus, la face autour des antennes et sous ces dernières, les joues et la région postérieure ;

- sur le thorax : les pleures;

- sur l'abdomen : la totalité du tergite 1, excepté une courte bande longitudinale médiane, deux fois plus étroite que le scutellum; la plus grande partie du tergite 2, excepté une large zone médiodorsale triangulaire, dont la base longe le bord du tergite 2 et dont la pointe atteint-łe tergite 3 ; le bord postérieur des tergites 3 à 6 , constituant ainsi un liséré comme chez I. marginata: la totalité des sternites. 
Genitalia.
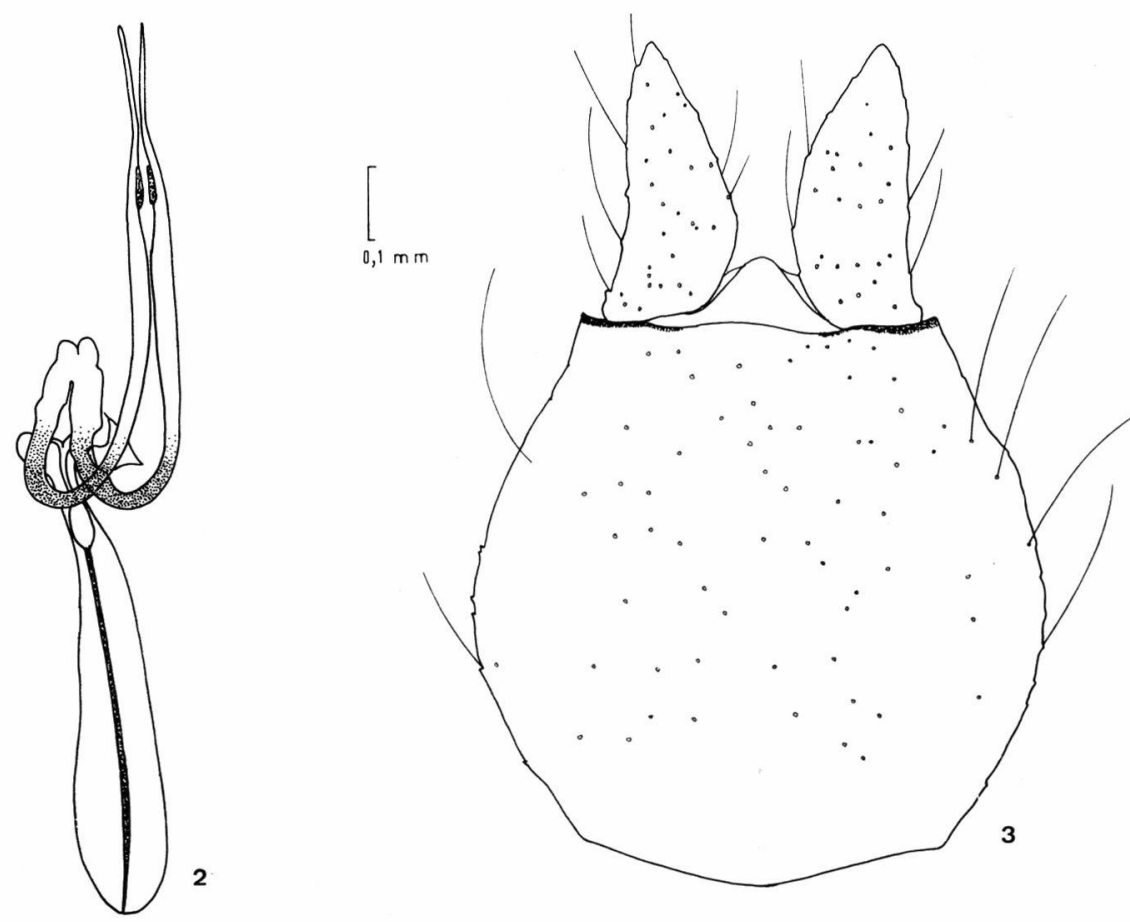

Fig. 2-3. - Ibisia vaillanti. - 2 : vue dorsale oblique de l'edeagus ; 3 : vue dorsale de l'epandrium et des cerques (holotype, Haute-Garonne).

Ils sont brun noirâtre et de morphologie voisine de ceux d'I. marginata ; en particulier, les édéages des deux espèces paraissent pratiquement identiques. Malgré une certaine variabilité (fig. $4 \mathrm{a}, \mathrm{b}, \mathrm{c}$ et 3 ), les cerques sont plus longs que chez I. marginata (fig. 5) et ont un contour interne plus régulièrement arrondi. Les dististyles sont un peu plus massifs à la base et à l'apex chez $I$. vaillanti. Principal caractère distinctif, net et constant $:$ le fort élargissement du basistyle, près de l'apex, côté interne, de part et d'autre de la région terminale du pénis, chez $I$. vaillanti (fig. 4 ). Il forme, chez cette espèce, une sorte de palette profonde qui atteint presque le pénis, alors que chez I. marginata (fig. 5), les bords interne et externe du basistyle sont subparallèles (voir aussi les fig. 34 de Rozkośný et Spitzer 1965 et 26 de Thomas 1974). Le basistyle est en outre plus arrondi, en coupe transversale, que chez I. marginata où il forme une crête longitudinale nette.

Taille.

Longueur de l'aile :

- exemplaires français : 6,9 à $7,3 \mathrm{~mm}$, donc très comparable à celle 


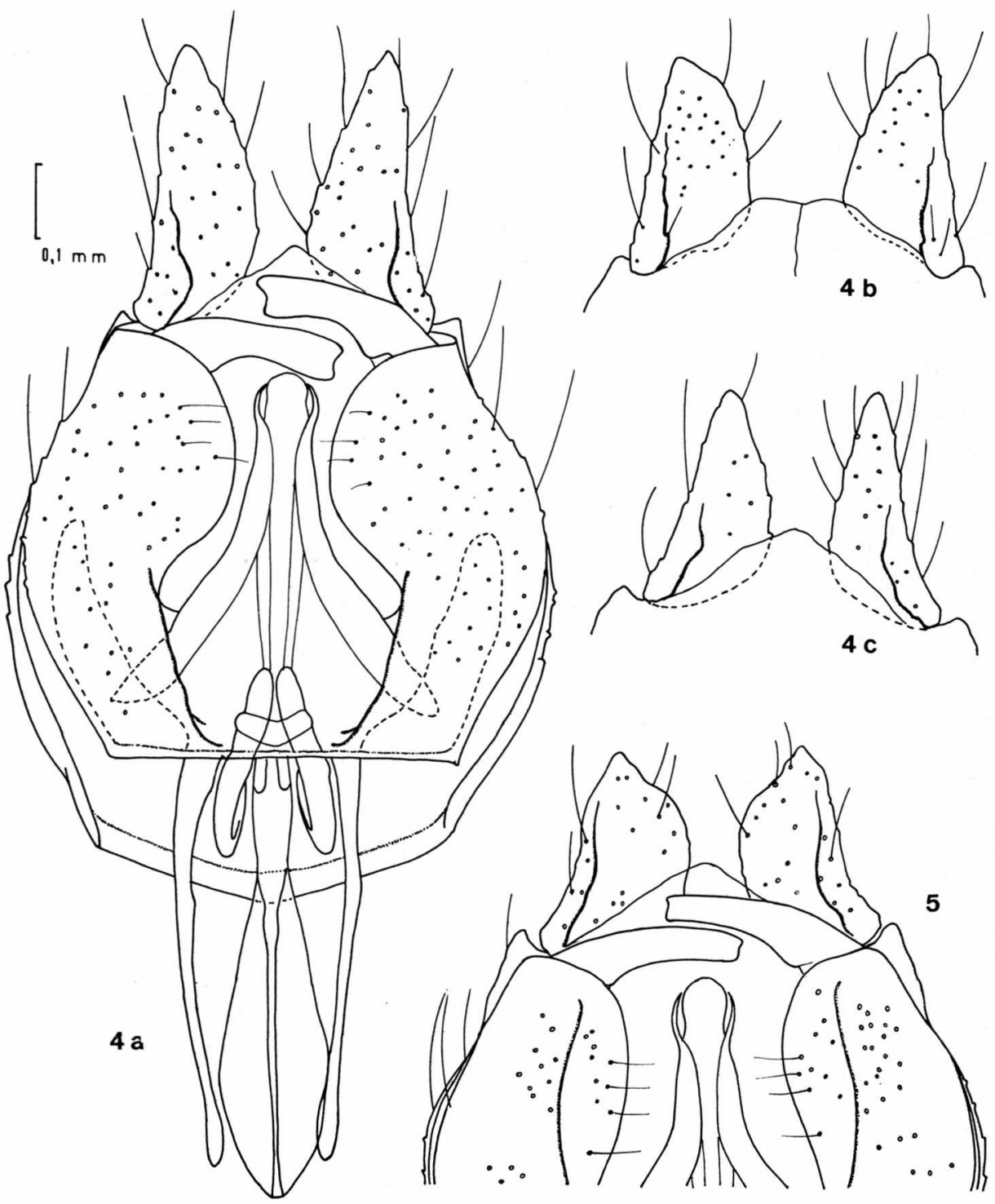

Fig. 4. - Ibisia vaillanti (vues ventrales). - $4 \mathrm{a}$ : genitalia (holotype); $4 \mathrm{~b}$ et $4 \mathrm{c}$ : variations de la morphologie des cerques $(4 \mathrm{~b}$ : spécimen de l'Ardèche, $4 \mathrm{c}$ : spécimen de Galice).

FIg. 5. - Ibisia marginata. - Genitalia en vue ventrale partielle. 
des imagos d'I. marginata des mêmes régions (Thomas 1974);

— exemplaires espagnols : 6,6 à $7,1 \mathrm{~mm}$.

\section{IMAGo ?.}

Les $q$ sont difficilement séparables de celles d'I. marginata. Les caractères distinctifs des genitalia que j'ai pu observer ne me paraissent pas constants. Cette étude sera reprise sur un matériel plus abondant.

MATÉRIEL EXAMINÉ.

12 ô provenant des localités suivantes :

\section{- France :}

Marcols à $700 \mathrm{~m}$ (Ardèche), élevage, V-1967, 1 of (F. Vaillant leg.); ia Truyère à $930 \mathrm{~m}$ (Lozère), 28-VI-74, 1 of; le Lot à $350 \mathrm{~m}$ (Aveyron), 10-VII-73, 1 \& ; le Laudot à $480 \mathrm{~m}$ (Tarn), élevage, VI-72, 1 o ; la Louge à $190 \mathrm{~m}$ (Haute-Garonne), 18-VII-72, 1 ô (holotype); le Nistos à $470 \mathrm{~m}$ (Hautes-Pyrénées), 8-VIII-68, $1 \hat{o}$; le ruisseau de Thuir d'Evol à $850 \mathrm{~m}$ (Pyrénées-Orientales), 12-VII-75, 2 ^̊.

\section{- Espagne :}

Le Rio Tambre à 230 et $280 \mathrm{~m}$ (Galice), 21-VII et 10-VIII-79, 4 s (E. Martinez leg.).

Deux paratypes sont déposés au Muséum de Paris.

Cette espèce est dédiée à $M$. le Professeur F. Vaillant (Grenoble) qui a capturé le premier spécimen de cette collection. Je le remercie cordialement, ainsi qu'E. Martinez (Orense).

En Europe Occidentale, le genre Ibisia n'est donc pas représenté par la seule espèce marginata - décrite il y a deux siècles —, comme on le croyait jusqu'ici (Thomas 1978). La liste des stations de récolte montre que les deux espèces $I$. vaillanti et $I$. marginata peuvent éventuellement cohabiter ( $R$. de Laudot). Mais il semblerait qu'I. vaillanti soit plus potamophile qu'I.marginata - surtout inféodée aux ruisseaux étroits et ombragés -, ou tout au moins, qu'elle s'accommode d'eaux plus chaudes et de lits plus larges. 


\section{TRAVAUX CITÉS}

Rozkośný (R.) et SPITZer (K.). 1965. - Schnepfenfliegen (Diptera, Rhagionidae) in der Tschechoslowakei. Acta ent. bohemoslov., 62 : 340-368.

Thomas (A. G. B.). 1974. - Diptères torrenticoles peu connus: I. Les Athericidae (larves et imagos) du Sud de la France (Brachycera, Orthorrhapha). Annls Limnol., 10 (1) : 55-84.

Thomas (A.). 1978. - Athericidae et Rhagionidae. In J. Illies: Limnofauna Europaea. Fischer Verlag, Stuttgart. XVII +532 p. 